\title{
Observational aspects of gamma-ray burst afterglows
}

\author{
Thomas Krühler* \\ Dark Cosmology Centre Niels Bohr Institute, University of Copenhagen, Juliane Maries Vej 30, \\ 2100 Copenhagen Denmark \\ E-mail: tomedark-cosmology.dk
}

For our understanding of the physics of GRB explosions as well as their implications on star formation and cosmology, it is crucial to observe the luminous afterglow emission. Optical/nearinfrared afterglow observations provide accurate physical scales to GRBs, and allow us to put constraints on the properties of the ultra-relativistic outflow. In the recent years, systematic follow-up campaigns have led to an increasing sample of high-quality afterglow light-curves, multi-wavelength SEDs and spectra. This new data shed light on the properties of the complete population of long GRBs, including those events that previously have escaped detection. I will give an overview of the state-of-the-art of the field and highlight new observational results.

Gamma-Ray Bursts 2012 Conference-GRB2012,

May 07-11, 2012

Munich, Germany

${ }^{*}$ I am very grateful to J. Greiner, R. Filgas, D. A. Kann, D. Malesani, A. Rau, P. Schady, M. Nardini, J. P. U. Fynbo, S. Savaglio, S. Klose, S. McBreen, A. Rossi, J. Elliott and many more for insightful discussion

†Speaker. 


\section{Introduction}

Ultra-violet/optical/near-infrared (NIR) observations of the afterglow of long gamma-ray bursts (GRBs) are a versatile probe of GRB physics. They yield not only the most reliable and direct measurements of the redshift of GRBs, thus setting the physical scales, but allow us also to constrain emission models, the jet composition and geometry and the circumburst medium. These observations are thus fundamental to reliably test central engine or progenitor scenarios and crucial to robustly use GRBs as tools for tracing high-redshift galaxies [12, 26], cosmic-chemical evolution [24] and the star-formation history of the Universe [3].

The recent years have seen a tremendous increase in the quality and quantity of optical/NIR afterglow data, primarily thanks to the rapid localization and follow-up capabilities of the Swift satellite, the advent of (quasi)-robotic and dedicated telescopes with apertures of up to $\sim 2 \mathrm{~m}$ and comprehensive spectroscopic campaigns on the worlds largest telescopes. Equipped with automatic scheduling that yield an immediate response to GRB triggers as well as efficient instruments, these instruments provide high-quality data that can be used to study a larger sample of individual afterglows in unprecedented detail. These data are furthermore the basis of afterglow samples without significant physical biases in, for example, the redshift distribution of GRBs or properties of GRB hosts.

Exemplary of this new class of instruments is the Gamma-Ray burst Optical/Near-infrared Detector (GROND, [8]), mounted at the $2.2 \mathrm{~m} \mathrm{MPI/ESO} \mathrm{telescope} \mathrm{on} \mathrm{LaSilla.} \mathrm{With} \mathrm{its} \mathrm{very}$ rapid ( $\gtrsim 100 \mathrm{~s}$ ) response to GRB triggers, and simultaneous imaging capabilities in seven filters $\left(g^{\prime} r^{\prime} i^{\prime} z^{\prime} J H K_{s}\right)$ from the optical to the NIR, GROND gives observational access to faint afterglows that previously escaped detection due to intrinsic faintness, a high redshift, or substantial amount of dust along the sight-line to the GRB.

\section{The bright end of the afterglow luminosity distribution}

\subsection{The afterglows of Fermi/LAT GRBs}

Fermi can measure the spectral properties of gamma-ray bursts over a very large energy range and is opening a new window on the prompt emission characteristics of GRBs. The GRBs detected by the Large Area Telescope (LAT) up to energies of several GeV are among the most energetic GRBs with isotropic-equivalent energies emitted in $\gamma$-rays $E_{\gamma, \text { iso }}$ approaching $10^{55} \mathrm{erg}$ [10].

The optical/NIR afterglows of these extremely energetic Fermi GRBs are bright and luminous observationally as well. They lie in the upper half of the brightness distribution of all optical afterglows detected in the Swift era or even in the top 5\% if incompleteness in the Swift afterglow sample is considered [18, 22]. The properties of the host galaxies of LAT detected bursts in terms of extinction, star formation rates and masses do not appear to differ from previous samples of long GRB hosts [18].

The complete (emission-angle corrected) kinetic energy budget of those events can pose strong challenges on emission models. The available relativistic energy from a magnetar-powered GRB for example is expected to be lower than $E_{\text {kin }}<10^{51} \mathrm{erg}$. This limit is violated by at least some of the events [2], whereas the energy budget of even the most energetic Fermi GRBs is consistent with 


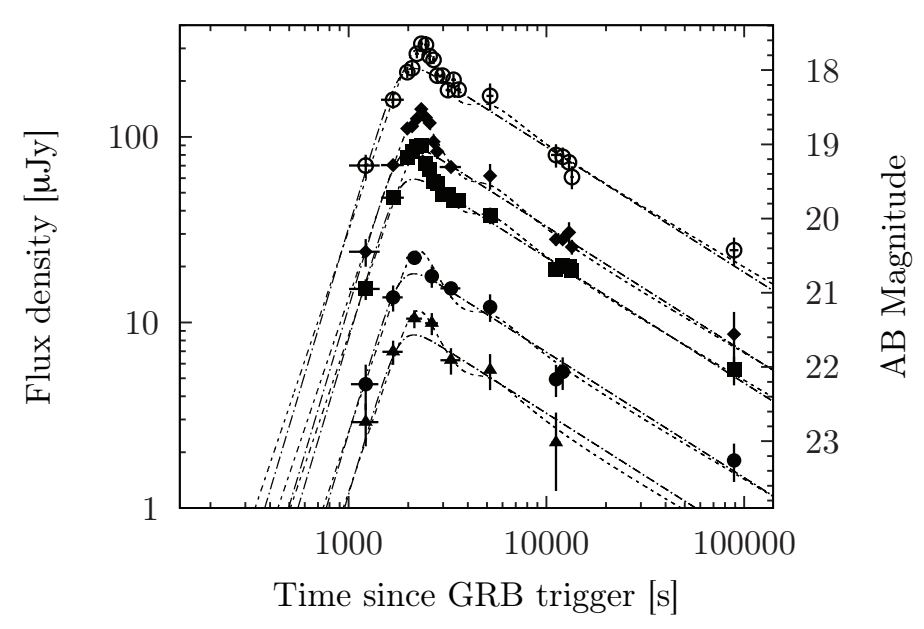

Figure 1: Optical/NIR light-curve of the afterglow of GRB 070802. Five different filters from the GROND instrument $\left(r^{\prime} z^{\prime} J H K_{s}\right)$ are shown, illustrating the achromatic nature of rise and decay. Adapted from [14].

Collapsar models. Here, the gravitational energy of a rotating black hole - accretion disk system is converted into jets via e.g., the Blandford-Znajek mechanism, or neutrino annihilation.

\subsection{The onset of the afterglow}

The optical/NIR afterglow light curve is in many cases dominated by an early increase in brightness [19]. This early rise is achromatic (see Fig. 1). Thus a movement of the characteristic synchrotron frequency through the observed optical bands, as well as dust destruction are readily ruled out as the origin of the initial rise [16,21]. The observational characteristics of this afterglow onset are a temporal rise of $\propto t^{0.5-3}$ and a very smooth turnover to the subsequent decay (see Fig. 1).

The early rise in the optical afterglow light-curve is generally attributed to the onset of the forward shock emission. This happens when the swept up medium efficiently decelerates the ejecta. From the time of the light curve peak (typically at few tens to hundreds of seconds), physical parameters of the outflow, such the deceleration radius $r_{\mathrm{dec}}$ and the Lorentz-factor $\Gamma_{\mathrm{dec}}$ at $r_{\mathrm{dec}}$ can be constrained. $\Gamma_{\text {dec }}$ is a very good proxy of the initial bulk Lorentz-factor $\Gamma_{0}$ as $\Gamma_{0} \sim 2 \times \Gamma_{\text {dec }}$. The early optical afterglow hence provides a robust measurement and confirmation of the ultrarelativistic nature of the GRB phenomenon, yielding values of $100 \lesssim \Gamma_{0} \lesssim 500$, and deceleration radii of $10^{16}$ to $10^{17} \mathrm{~cm}$ with only a weak dependence on the uncertain micro-physical parameters.

Even higher Lorentz-factors approaching or possibly exceeding $\Gamma_{0} \sim 1000$ arguably exist. Optical follow-up observations are in those cases however not rapid enough to catch the peak of the afterglow emission at a few seconds after the initial $\gamma$-ray trigger.

\subsection{Variability in optical light-curves}

Among the diversities in the very early evolution of GRB afterglows are bright optical/nearinfrared flares before or superimposed onto an otherwise smoothly decaying afterglow light curve.

In some cases, early optical observations reveal a light-curve morphology that is remarkably similar to the X-ray flares, which are detected in approximately $50 \%$ of all X-ray afterglows. The 


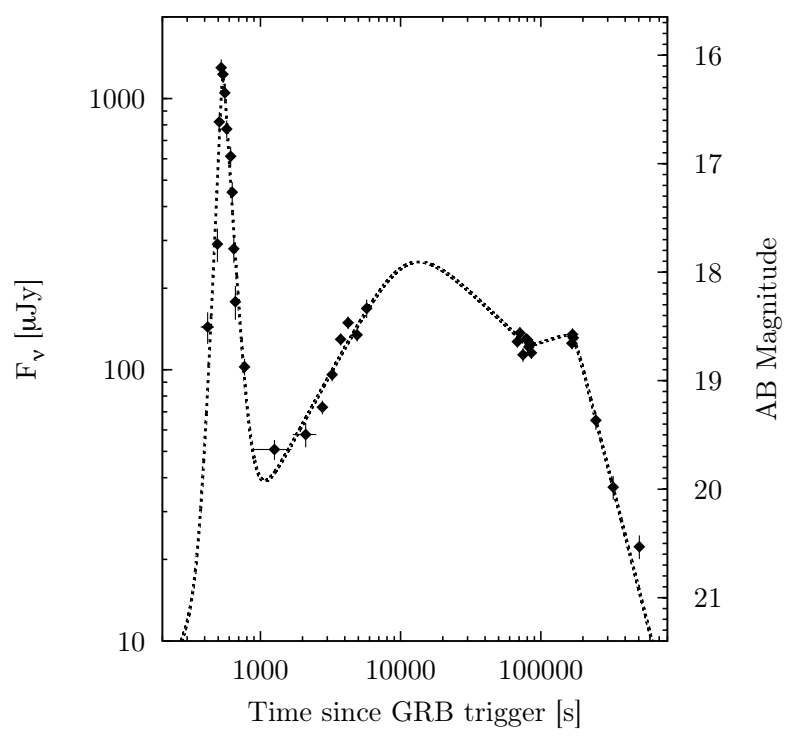

Figure 2: The optical flare of GRB 080129 before its rising afterglow as obtained with the Gamma-ray Optical Near-infrared Detector (GROND). The light-curve is parametrized with a series of smoothly connected power-laws (dashed lines). Super-imposed to the overall afterglow evolution peaking at $10 \mathrm{ks}$, a strong flare with fast rise and fast decay at $t \sim 500 \mathrm{~s}$ is apparent. Adapted from [9].

morphology of the flares is characterized by a very fast rise and similarly fast decay (see Fig. 2). Furthermore, optical flares show strong spectral evolution and time lags, and are in many cases correlated with contemporaneous X-ray flares. There can also be multiple optical/NIR flares for a single event [15].

This strongly suggests late central engine activity as the common origin. The optical/NIR flares with fast rise and fast decay would thus be the soft tail of emission correlated with late internal shocks. This connection provides additional evidence that inner engine activity may last or be revived on a timescale of hours or days at least for some bursts.

The early optical afterglow light curve is however not as often and not as strongly affected as the X-rays by flaring episodes. This is readily explained with the spectrum of the flares (peaking in the sub $\mathrm{keV}$ to few $\mathrm{keV}$ range), and a bright forward shock component typically dominating the optical emission. Thus, if the emission in the flares is not strong enough with respect to the underlying forward-shock emission, a bright afterglow can easily outshine flare signatures in the optical bands even for very luminous X-ray flares.

\subsection{Decoupled multi-wavelength light-curves}

There is a growing number of afterglows with well-sampled multi-wavelength afterglow light curves that start very early $(t \sim 100 \mathrm{~s})$ after the trigger. While some of the events show the characteristic observational signatures expected from a simple external-shock jet model such as a forwardand reverse-shock, correlated optical/X-ray decays and jet-breaks [7], a significant number of wellobserved GRB afterglows is inconsistent with the simplest fireball scenarios $[4,5]$. 


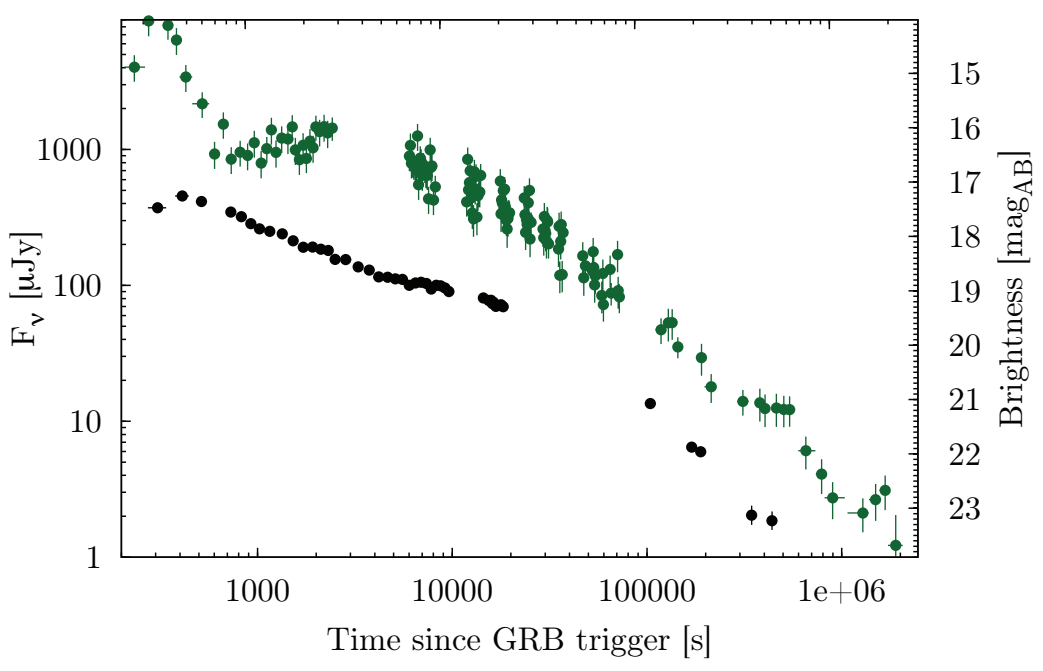

Figure 3: The decoupled optical $r^{\prime}$-band (black) and X-ray (green) afterglow of GRB 091029 [6].

An extreme example of virtually fully decoupled optical/NIR and X-ray light-curves is shown in Fig. 3, making the afterglow of GRB 091029 difficult to explain in the framework of the basic fireball model. Instead it provides evidence for two different emission mechanisms dominating the different energy ranges [6]. Some of the decoupled lightcurves could potentially be explained by various extensions to the fireball model, such as two-component jet models, outflows observed off-axis, (chromatic) energy injections or temporal variations of the micro-physical parameters.

The complexity and number of free parameters of these modified fireball models, however, can be so high, that even state-of-the-art data does not test these models robustly [6]. Possibly, alternative models need to be pursued in the future to account for an increasing variety and decoupling in the morphology of optical/NIR and X-ray afterglow light curves.

\section{Afterglow observations in the sample era}

As redshift measurements of GRBs typically require an optical afterglow, there are substantial selection biases due to the phenomenon of optically-dark GRBs. A high redshift and significant column densities of dust along the GRB's sight line efficiently suppress afterglow emission in the UV/optical wavelength bands, where most of the follow-up observations and redshift measurements are performed. This bias is only resolved through samples with high completeness in redshift $[11,12]$. This is achieved by making use of the brighter afterglow emission at earlier times, or host spectroscopy in those cases where redshifts can not be obtained via afterglow spectroscopy $[13,17]$.

\subsection{The nature of dark GRBs}

Once samples with high redshift completeness are available, it is straight forward to infer the nature of dark GRBs, where the optical afterglow is suppressed with respect to the emission in the $\mathrm{X}$-ray regime. Based on the highly complete GROND sample, two main contributing factors to the 


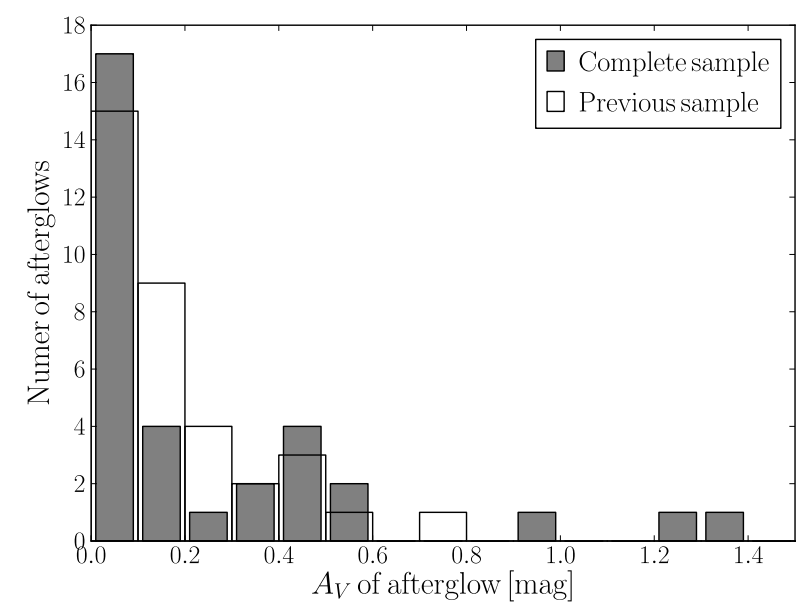

Figure 4: The distribution of measured $A_{V} \mathrm{~s}$ from the sample of [11] compared to values from the literature.

darkness of afterglows were identified [11]: (i) moderate intrinsic extinction at moderate redshifts, and (ii) about $1 / 5$ of dark bursts at redshift $z>5$. Similar conclusions were obtained based on the afterglow sample from the P60 [1] in combination with host follow-up observation [20].

Taking this analysis one step further, one can use the multi-wavelength afterglow data to directly measure the amount of visual extinction $A_{V}$ along the sight line to the GRB. While previous sight lines towards GRBs did not contain a lot of dust $\left(A_{V} \lesssim 0.5\right)$, more complete samples show substantially larger $A_{V}$ values, with a $20-30 \%$ of all events at moderate $\left(A_{V} \sim 0.5\right)$ and $10-20 \%$ at high visual extinctions $A_{V} \gtrsim 1$. Values of $A_{V} \sim 3-5$ have been observed via NIR follow-up observations for some very red events, and even higher visual extinctions arguably exist as well. Despite this larger fraction of moderate to large extinction, there is still a 40-50\% fraction of bursts with $A_{V} \sim 0$ (Figure 4).

\subsection{The fraction of high-redshift GRBs}

Afterglow samples also provide the best measurements currently available for the fraction of high- $z$ GRBs. Different recent works yield consistent results of $5.5 \pm 2.8 \%(z>5)[11],<14 \%$ $(z>5),<7 \%(z>7)$ [20], $3-5 \%(z>5)[23]$ or $<14 \%(z>5),<5 \%(z>7)$ [13].

Although the redshift distribution of GRBs is clearly not dominated by $z>5$ events (median redshift of Swift GRBs is $z=2.1$ ), these fractions are still two orders of magnitude higher than for Quasars selected from the SDSS and UKIDSS, for example. GRBs remain thus primary targets to investigate the high-redshift Universe in unprecedented detail, and the highest spectroscopicallyconfirmed redshift for any astronomical object known to date is still GRB 090423 at $z=8.2$ [25].

\subsection{The fraction of star-formation as traced by GRBs}

A sample that is complete in redshift is also the basis for deeper insights into the relation of global star-formation rate (SFR) and GRB rate $\left(\rho_{\mathrm{GRB}}\right)$, typically parametrized with a power-law dependence in redshift $\left(\rho_{\mathrm{GRB}} \propto(1+z)^{\alpha} \times \mathrm{SFR}\right)$. A firm understanding of this relation, coupled with the high-redshift nature of GRBs would then facilitate an investigation of the SFR beyond what 
is currently possible with Lyman-break galaxies or $\operatorname{Ly} \alpha$ emitters. GRBs also have the advantage that their selection is independent on dust and galaxy luminosity, avoiding some of the most severe selection biases in high-redshift galaxy studies.

Although the largest uncertainty, the effect of dark bursts, has been minimized through the available new afterglow samples, no consensus about $\alpha$, the redshift dependence between GRB rate and SFR has been reached in the literature [23, 13,3]. Current estimates range from no or extremely weak $(\alpha \sim 0)$ up to a highly significant $(\alpha \sim 2)$ dependence. Arguably, a better understanding of the GRB luminosity function, and larger number statistics of redshift-complete GRB samples will play a crucial role in resolving this discrepancy in the future.

\section{Summary and Outlook}

The increase in data quality and quantity due to dedicated GRB follow-up programs with sophisticated instrumentation on medium-to-large aperture telescopes has allowed us to measure the Lorentz factor of the ultra-relativistic outflow for the first time in larger samples, revealed strong variability in a significant fraction of all optical afterglows, and has constrained possible scenarios for the central engine via the ultra-luminous Fermi/LAT GRBs and afterglows. It furthermore provided evidence for complex emission mechanisms much beyond the very simple, jetted synchrotron shock model. These complex models are required to account for the observations of highly decoupled optical and X-ray light curves.

Studies of individual events, primarily taken from the bright end of the afterglow luminosity distribution are now being complemented by GRB research via well-defined samples with number statistics larger than 40 and a high redshift completeness. Ideally, these samples are representative of the complete parameter space of GRBs (and thus include the correct proportion of faint, dustextinguished and high-redshift events) and are not biased by optically dark bursts.

Once the initial selection function of GRBs is understood, samples are sufficiently large and redshifts are measured for a large fraction ( $\gtrsim 80-90 \%$ ) of the selected GRBs (either through afterglow of host spectroscopy), these samples can be used for an unbiased census of GRBs, their afterglows and hosts. They will thus give important insights into the star-formation history back into the epoch of re-ionization and the high-redshift population of star-forming galaxies.

\section{Acknowledgments}

Part of the funding for GROND (both hardware as well as personnel) was generously granted from the Leibniz-Prize to Prof. G. Hasinger (DFG grant HA 1850/28-1).

\section{References}

[1] Cenko, S. B. et al., Dark Bursts in the Swift Era: The Palomar 60 Inch-Swift Early Optical Afterglow Catalog, ApJ 693 (2009) 1484, [arXiv: 0808 . 3983]

[2] Cenko, S. B. et al., Afterglow Observations of Fermi Large Area Telescope Gamma-ray Bursts and the Emerging Class of Hyper-energetic Events, ApJ 732 (2011) 29 [arXiv: 1004.2900 ]

[3] Elliott, J., et al., The long $\gamma$-ray burst rate and the correlation with host galaxy properties, A\&A 539 (2012) 113, [arXiv:1202.1225] 
[4] Filgas, R., et al., The two-component jet of GRB 080413B A\&A 526 (2011)113 [arXiv: 1012 . 0328]

[5] Filgas, R., et al., GRB 091127: The cooling break race on magnetic fuel, A\&A 535 (2011) 57 [arXiv:1109.2810]

[6] Filgas, R., et al., GRB 091029: At the limit of the fireball scenario, A\&A, submitted (2012)

[7] Gendre, B. et al., GRB 110205A: Anatomy of a Long Gamma-Ray Burst, ApJ 748 (2012) 59 [arXiv:1110.0734]

[8] Greiner, J. et al., GROND - a 7-Channel Imager, PASP 120 (2008) 405 [arXiv:0801.4801]

[9] Greiner, J. et al., A Strong Optical Flare Before the Rising Afterglow of GRB 080129, ApJ 693 (2009) 1912 [arXiv:0811.4291]

[10] Greiner, J. et al., The redshift and afterglow of the extremely energetic gamma-ray burst GRB 080916C, A\&A 498 (2009) 89 [arXiv:0902.0761]

[11] Greiner, J. et al., The nature of dark gamma-ray bursts, A\&A 526 (2011) 30 [arXiv: 1011.0618 ]

[12] Hjorth, J., et al., The TOUGH survey: I. Survey design and catalogs, ApJ, submitted (2012) [arXiv:1205.3162]

[13] Jakobsson, P., et al., The TOUGH survey: III. Redshift Distribution, ApJ, in press (2012) [arXiv:1205.3490]

[14] Krühler, T. et al., The 2175 ÅDust Feature in a Gamma-Ray Burst Afterglow at Redshift 2.45, ApJ 685 (2008) 376 [arXiv:0805.2824]

[15] Krühler, T. et al., Correlated Optical and X-Ray Flares in the Afterglow of XRF 071031, ApJ 697 (2009) 758, [arXiv:0903.1184]

[16] Krühler, T. et al., The bright optical/NIR afterglow of the faint GRB 080710 - evidence of a jet viewed off-axis, A\&A $\mathbf{5 0 8}$ (2009) 593 [arXiv: 0908 .2250]

[17] Krühler, T. et al., The TOUGH survey: V. VLT/X-shooter emission-line redshifts for Swift GRBs at $z \sim 2$, ApJ submitted (2012) [arXiv: 1205.4036]

[18] McBreen, S. et al., Optical and near-infrared follow-up observations of four Fermi/LAT GRBs: redshifts, afterglows, energetics, and host galaxies, A\&A 516 (2010) 71 [arXiv: 1003.3885 ]

[19] Molinari, E. et al., REM observations of GRB 060418 and GRB 060607A: the onset of the afterglow and the initial fireball Lorentz factor determination, A\&A $\mathbf{4 6 9}$ (2007) 13 [arXiv:astro-ph/0612607]

[20] Perley, D. et al., The Host Galaxies of Swift Dark Gamma-ray Bursts: Observational Constraints on Highly Obscured and Very High Redshift GRBs, AJ, 138 (2009) 1690 [arXiv: 0905.0001$]$

[21] Perley, D. et al., Evidence for supernova-synthesized dust from the rising afterglow of GRB071025 at $z \sim 5$, MNRAS 406 (2010) 2473 [arXiv: 0912 . 2999]

[22] Rau, A. et al., A Very Metal-poor Damped Lyman- $\alpha$ System Revealed Through the Most Energetic GRB 090926A ApJ 720 (2010) 862 [arXiv: 1004 . 3261]

[23] Salvaterra, R., et al., A Complete Sample of Bright Swift Long Gamma-Ray Bursts. I. Sample Presentation, Luminosity Function and Evolution, ApJ 749 (2012) 68 [arXiv: 1112 . 1700 ]

[24] Savaglio, S., et al., Supersolar metal abundances in two galaxies at $z \sim 3.57$ revealed by the GRB 090323 afterglow spectrum, MNRAS 420 (2012) 627 [arXiv: 1110 . 4642] 
[25] Tanvir, N. R., et al., A $\gamma$-ray burst at a redshift of $z \sim 8.2$, Nature 461 (2009) 1254 [arXiv:0906.1577]

[26] Tanvir, N. R., et al., Star formation in the early universe: beyond the tip of the iceberg, ApJ in press, (2012) [arXiv:1201.6074] 